\title{
Reliability Assessment of AC/DC Hybrid Distribution Network Based on Sequential Monte Carlo Method
}

\author{
Wang Ziyi ${ }^{1}$, Yang Xiaomin, Xiao Huixu, Chen Hongtao \\ ${ }^{1}$ Beijing Jiaotong University,Haidian District, Beijing ,China
}

Keywords: AC/DC hybrid distribution network, reliability assessment, minimal path sets, sequential Monte Carlo method

\begin{abstract}
Traditional AC distribution network usually adopts "closed loop design, open loop operation" mode of power supply, which restricts the further increase of the power supply reliability. When the DC network joins in, it is possible to realize the closed loop operation through reasonable control strategies, so as to improve the distribution network reliability. This paper mainly studied the operation mode and its reliability assessment of the AC/DC hybrid distribution network. Two-terminal power supply distribution structure was selected to be transformed to AC/DC hybrid distribution network containing flexible HVDC. Then the system control and operation mode of the new distribution model was studied and discussed. Adopting sequential Monte Carlo method based on the minimal path sets, the reliability of AC/DC hybrid distribution network was assessed and compared with traditional AC distribution network according to the cases.
\end{abstract}

\section{Introduction}

With the emergence of new problems caused by large-scale renewable energy and DC loads connected to the grid, and the maturity and development of the power electronics and flexible HVDC technology, DC network accessed to distribution network is getting more and more attention at home and abroad. In the traditional AC power distribution network "ring network design, open loop operation" mode, the regional contact switch normally in a disconnected state, limited to further improve the reliability of power supply[1]. In recent years, with the development of distribution network, flexible DC transmission is not limited by the electromagnetic loop network and able to control the flow, so in the distribution network can simultaneously access multiple AC power, to achieve closed-loop operation, thereby improving power supply reliability[2-4]. So it is very important to evaluate the reasonable reliability of AC / DC hybrid distribution network.

[5]focuses on the "hand in hand" structure of the AC distribution network and DC distribution network, using the minimum cutting set of the two distribution network reliability of the comparative analysis, pointed out that under the conditions of the development of existing technology the reliability of the DC distribution network is actually lower than that of the AC distribution network. [6]-[7]using a non-sequential Monte Carlo simulation to complete the 
reliability of the entire system evaluation,[8]using F \& D-Monte Carlo hybrid method to simulate and calculate the reliability index of AC / DC hybrid system

In the reliability evaluation of DC distribution network, low voltage DC distribution grid and DC micro-grid are mainly concerned at home and abroad[9-11], research of adding flexible DC distribution line to the medium voltage $\mathrm{AC}$ distribution network to constitute $\mathrm{AC} / \mathrm{DC}$ hybrid system is less. To solve the problems, this paper studies the typical AC/DC hybrid power distribution network operation mode. And fault analysis is made. In order to study the reliability improvement of AC / DC hybrid distribution network, the reliability model of AC / DC hybrid distribution network system is obtained. Adopting sequential Monte Carlo method based on the minimal path sets, the reliability of AC/DC hybrid distribution network was assessed. According to the evaluation results analysis to make a conclusion and provide feasible suggestions for the practical application of AC/DC hybrid power distribution network.

\section{Operation mode and fault analysis of AC / DC hybrid power distribution network}

Considering the medium voltage flexible DC distribution networks, this paper takes AC/DC hybrid distribution network as the research object, in which DC network is connected to the twoterminal power supply structure AC distribution, its structure is shown in Figure 1. The twoterminal types of AC/DC hybrid power distribution network are mainly composed of $10 \mathrm{kv}$ AC bus and $\pm 10 \mathrm{kv}$ DC bus and DC lines. Among them, the DC bus connected to the AC power supply bus through the VSC converter. Wind power, photovoltaic and energy storage devices, as well as AC and DC load through the two DC transformers access to DC power distribution lines.

In the normal operation mode, $\mathrm{AC} / \mathrm{DC}$ hybrid distribution network mainly regard the large power grid as a DC distribution network energy and voltage support. The active power between the converter station and the $\mathrm{AC}$ grid and between the distributed power supply and the DC bus must be evenly distributed, ie the active power flow entering the DC power line must be balanced and any difference will cause the system's DC voltage to rise or fall rapidly. To achieve this balance, one of the converter stations must use constant DC voltage control.

According to the above principles, the operation mode of this study with flexible DC power distribution line hybrid AC / DC power distribution system is analyzed (Figure 1). AC / DC hybrid system can switching operation mode by controlling the system, there are 4 main operating mode: two-terminal power supply, single terminal power supply, two-terminal isolation, island operation.

Consider the operation mode control of bidirectional VSC, do the following analysis and treatment to the fault:

1) When the fault is in the AC branch line (AC circuit, AC circuit breaker, AC isolation switch), directly accumulate the number of failures, power outage time.

2) When one of the VSC1 or VSC2 faults, the control switch to the operating mode 2 (VSC1 fault, by the AC side 2 for the DC distribution network single-ended power supply; VSC2 fault, by the AC side 1 for the DC distribution network single-ended power supply);

3) When the DC bus or DC circuit breaker failure, control switch to the operating mode 3;

4) When the VSC1 and VSC2 fault at the same time, control switch to the operating mode 4;

5) When the fault occurs in the DC branch line (DC line, DC circuit breaker, DC isolation switch, distributed power supply, energy storage, converter), directly accumulate the number of failures, power outage time. 


\section{Reliability evaluation of AC / DC hybrid distribution network}

\subsection{Reliability assessment process based on sequential Monte Carlo}

This paper selects sequential Monte Carlo method to evaluate reliability of DC distribution network. When the fault scene is determined, the fault analysis method based on the minimum path is used to analyze the fault results. The concrete steps are described as follows:

(1)Read the original data into the system.

(2)Set the simulation life, initialize the simulation time, fault parameters and component status, etc.

(3)The normal operating time (TTF) and the fault time (TTR) of all the components are sampled, and the operating duration duration of each element in the simulated total time is sequentially arranged. At the same time generate fan, photovoltaic and load the timing sequence.

Element i:

$$
\begin{aligned}
& T_{T T F}^{i}=-\left(1 / \lambda_{i}\right) \cdot \ln u \\
& T_{T T R}^{i}=-\left(1 / \mu_{i}\right) \cdot \ln u
\end{aligned}
$$

Where $\lambda_{i}$ and $\mu_{i}$ are the failure rate and repair rate of element $\mathrm{i} ; u$ is a random number that is uniformly distributed between $(0,1)$.

(4)Find the smallest element of TTF as the faulty element, analyze the fault event, and use the minimum road method to traverse the search process to find the load affected by the fault.

(5)For the load that can be restored by switching the operating mode, add the outage to the total blackout time $T_{\text {TTTR }}^{l}$ and the total number of blackouts $N_{l}$ of the load point,define $T_{\text {TTTR }}^{l}=T_{\text {TTTR }}^{l}+T_{\text {TMS }}$, $N_{l}++$, of which $T_{T M S}$ is the operational time of the operation mode switching.

For a load that can not be restored by transfer, the system's fault time is the load outage time. Define $T_{T T T R}^{l}=T_{\text {TTTR }}^{l}+T_{\text {TTRk }}, N_{l}++$, of which $T_{\text {TTRk }}$ is the system's fault time.

(6)Judge whether the current simulation time is greater than the preset time, if so, then the next step, or go to step (3)

(7)According to the total outage time $T_{T T T R}^{l}$ and the total number of power outages $N_{l}$ of each load point, calculate the average failure rate $\lambda_{l}$ of each load point, the average power outage time $r_{l}$, the average power failure time $U_{l}$ and other reliability indexes of the load points in the total simulation time.

(8)The reliability indexes of the system are calculated according to the reliability index of the load point.

\subsection{Failure consequence analysis based on the minimal path method in loop network operation}

In this paper, we use the search method in the minimum path method to traverse the component fault and search the impact load point. The basic idea of the search method is to start from the input node of the network to the output node in turn to find all the smallest path, this method is easy to achieve programming.

Because the DC distribution network is connected with the distributed power supply and the energy storage device, when the DC distribution network is in the island operation mode, the principle of the equipment processing on the minimal road is as follows: 
(1) If the equipment on the minimal path is in the island, then this kind of minimal path equipment failure will lead to the outage of the load point, participate in the reliability calculation is the equipment failure rate and repair time.

(2)If the equipment on the minimal path is outside the island, then this kind of minimal path equipment failure will lead to the outage of the load point

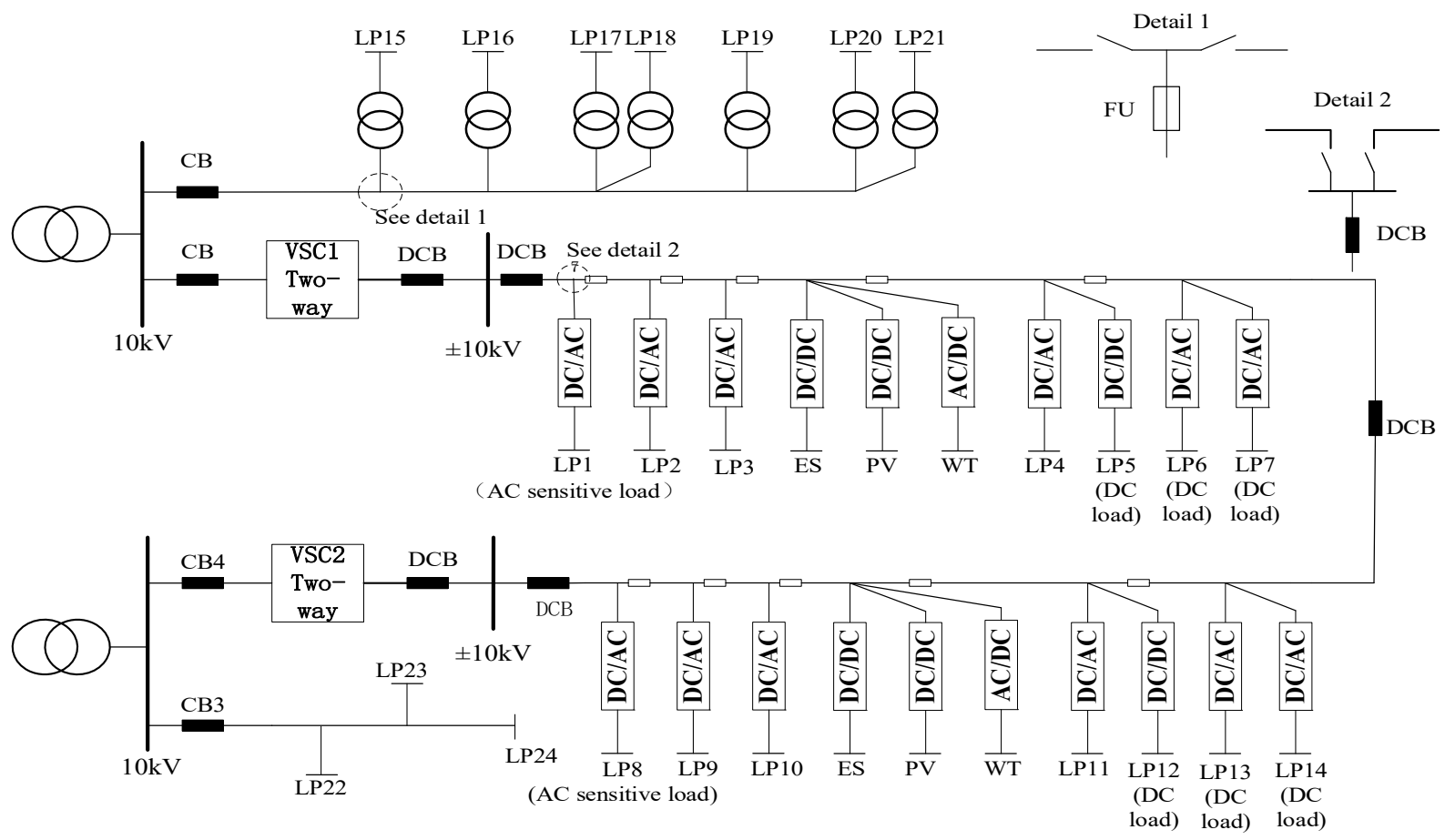

Figure.1 Modified IEEE RBTS BUS 4

\section{Example analysis}

This paper adopts modified IEEE RBTS Bus4 system as example, system architecture is shown in Figure 1, the feeder line between the F1 distribution line and F7 distribution line changed into DC line, which forms two-terminal power supply structure, to achieve the closed loop operation by DC circuit breaker. DC bus rated voltage of $\pm 10 \mathrm{kv}$, through the DC bus segment access AC load, DC load, photovoltaic, fan and energy storage. The system includes 24 load points, among which the $\mathrm{DC}$ load point is 6 , the $\mathrm{AC}$ load point is 18 , and the feeder in this example adopts the cable length, each feeder length is $0.7 \mathrm{~km}$, the specific data is shown in the table. PV and fan maximum output power of $1500 \mathrm{kw}$ and $2500 \mathrm{KW}$, respectively, storage capacity of $1000 \times 4 \mathrm{kwh}$. In order to analyze the advantages of $\mathrm{AC} / \mathrm{DC}$ hybrid distribution network compared with the traditional AC distribution network in terms of power supply reliability, the reliability evaluation method described in Section 3 of this paper is used to analyze the above example network and the traditional AC distribution network before transformation. The reliability indexes of the two systems are shown in Table 1. 
Table 1.Reliability Indexes of the Two Systems

\begin{tabular}{|c|c|c|c|c|}
\hline Power Supply Plan & $\begin{array}{c}\text { SAIFI } \\
\text { (Times/Year) }\end{array}$ & $\begin{array}{c}\text { SAIDI } \\
\text { (h/Year) }\end{array}$ & ASiAI & $\begin{array}{c}\text { EENS } \\
(\mathrm{mwh} / \text { Year })\end{array}$ \\
\hline Traditional AC Distribution Network & 1.3492 & 6.0050 & 0.9993 & 37.4760 \\
\hline C/DC Hybrid Distribution Network & 1.4129 & 4.6414 & 0.9995 & 23.9927 \\
\hline Compare Results & $1.017 \%$ & $22.708 \%$ & $0.016 \%$ & $-35.978 \%$ \\
\hline
\end{tabular}

Respectively for DC circuit breaker, transformer fault in DC converter station, the three kinds of DC equipment from the rate of the original parameters $20 \%, 40 \%, 60 \%, 80 \%, 100 \%, 120 \%$ for reliability assessment, obtained the average available rate of ASAI system is shown in Figure 2 respectively.

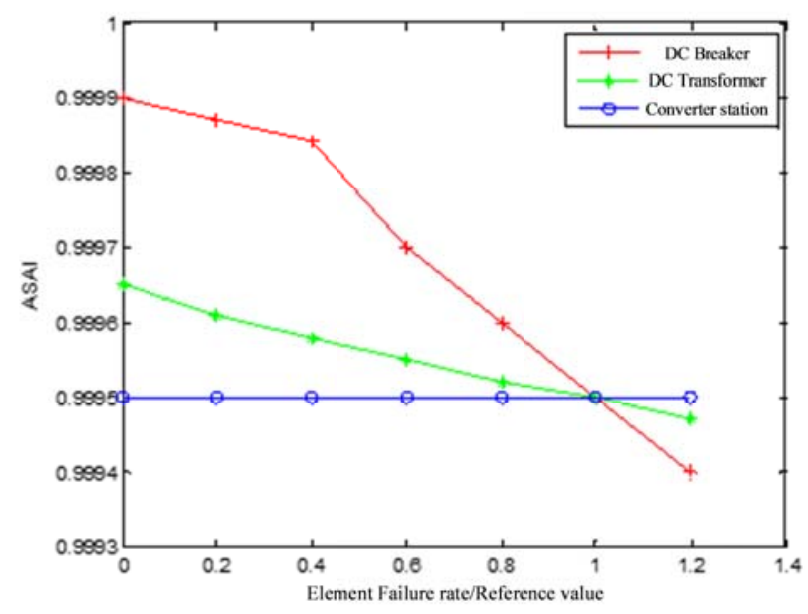

Figure 2. Sensitivity Analysis of the Effect of DC Component Failure Rate on the Reliability of AC / DC Hybrid Distribution Network

It can be seen from Table 1 and Figure 2, the SAIDI, ASAI, EENS three reliability indicators of AC/DC hybrid distribution network compared to the traditional AC distribution network have improved significantly, only SAIFI this indicator advantage is not obvious, this is because:

In the traditional AC distribution network, it is necessary to carry out the load transfer through the closed contact switch in the event of a fault, and the fault power failure time is longer after the contact switch operation time. The AC / DC hybrid distribution network is closed in normal operation mode. In the case of failure, the operation mode can be switched flexibly, so that the load can be transferred quickly, then the power outage time will be shorten greatly.

The distributed power supply of the traditional AC distribution network in the normal operation of the system are off-grid operation. In the AC/DC hybrid distribution network, the distributed power supply and energy storage are accessed to the grid under normal operating state. When the distribution network is switched to the island operation mode, the circuit breaker input time is not included, thus shortening the outage time of the load point in the island.

For the load of $\mathrm{AC}$ branch line, the power failure frequency is greatly reduced. However, due to DC elements used in the DC network are still in the research stage, the failure rate is high. Therefore, for the DC distribution line load, the power outage frequency is increased instead. The reliability index of the system reflects the overall reliability of the system. When the proportion of the load on the DC link is high, the average power failure frequency of the system will be worse. 


\section{Conclusion}

In this paper, through the analysis of the problem of "closed loop design and open loop operation" of traditional AC distribution network, it is suggested that the closed loop operation of AC distribution network can be realized by adding DC network, so as to improve the reliability of power supply. The distribution network of the two-terminal power supply structure is modified to form an AC/DC hybrid distribution network. The control and operation mode of the system are analyzed in detail. The reliability of the system is evaluated by using sequential Monte Carlo method based on the minimal path sets. It is proved that the $\mathrm{AC} / \mathrm{DC}$ hybrid distribution network can effectively improve the reliability of the distribution network. But because of the study of the DC circuit breaker and other key components is not yet mature, the failure rate is high. Thus the development of DC distribution network is limited. With the continuous development of power electronic technology and flexible HVDC technology applied in the power distribution, the performance of the DC component will reach a more ideal level, the failure rate will be reduced, as well as the power supply reliability of AC/DC hybrid power will be greatly improved.

\section{References}

[1] MA Long, YANG Jianhua, FANG Qin, SU Jian, WEI Tao, LIU Jun. Evaluation and Analysis of N-1 Criterion for Medium Voltage Distribution Network[J]. Electric Power Science and Engineering, 2013,02: 15-20.

[2] H. Shuai, J. Daozhuo, D. Yi and Y. Qunmin, "Research on the feasibility of ring structure of DC distribution network," Instrumentation and Measurement, Sensor Network and Automation (IMSNA), 2013 2nd International Symposium on, Toronto, ON, 2013, pp. 1075-1077.

[3] DOU Yi, JIANG Daozhi, YIN Rui, ZHENG Huan, WANG Yufen. Topology and Control Strategy of DC Distribution Network[J]. Electric Power Automation Equipment, 2015,01: 139-145.

[4] G. Li, G. Li, C. Zhao and H. Liang, "Research on Voltage Source Converter Based DC Distribution Network," 2007 2nd IEEE Conference on Industrial Electronics and Applications, Harbin, 2007, pp. 1927-1932.

[6] DING Ming, LI Xiao-yan, BI Rui, et al. Reliability evaluation of AC / DC hybrid transmission and transmission system with VSC-HVDC[J]. Power System Technology, 2008 (16): 53-58.

[7] XIE Shaoyu, WANG Xiu-li, WANG Xi-fan.Reliability Evaluation of AC / DC Hybrid System[J]. Electric Power Automation Equipment, 2011,31 (7): 10-16.

[8] TIAN Hui-fang. Reliability Evaluation of AC / DC Hybrid Power Grid Based on Hybrid Method[D]. North China Electric Power University (Baoding), 2012.

[9] Z. Ling, Y. Shan-shui, C. Lin and W. Li, "Reliability analysis of DC power distribution network based on minimal cut sets," Power Electronics and Applications (EPE 2011), Proceedings of the 2011-14th European Conference on, Birmingham, 2011, pp. 1-7.

[10] Stephen M. Frank, Steffen Rebennack, Optimal design of mixed AC-DC distribution systems for commercial buildings: A Nonconvex Generalized Benders Decomposition approach, European Journal of Operational Research, Volume 242, Issue 3, 1 May 2015, Pages 710-729

[11] Yong Jing, Xu Xin, Zeng Liqiang, Li Lulu. A Review of Research on Low Voltage DC Power Supply System[J]. Proceeding of the CSEE, 2013,07: 42-52 + 20. 